\title{
A JORDAN FACTORIZATION THEOREM FOR POLYNOMIAL MATRICES
}

\author{
H. K. WIMMER
}

\begin{abstract}
It is shown that a complex polynomial matrix $M(\lambda)$ which has a proper rational inverse can be factored into $M(\lambda)=\hat{C}(\lambda)(\lambda I-J) \hat{B}(\lambda)$ where $J$ is a matrix in Jordan normal form and the columns of $\hat{C}(\lambda)$ consist of eigenvectors and generalized eigenvectors of a linear operator associated with $M(\lambda)$. For a proper rational matrix $W$ with factorizations $W(\lambda)=$ $C(\lambda I-J)^{-1} B=M(\lambda)^{-1} P(\lambda)=Q(\lambda) N(\lambda)^{-1}$ it will be proved that $C$ consists of Jordan chains of $M$ and $B$ of Jordan chains of $N$.
\end{abstract}

1. Introduction. Any linear polynomial matrix $L(\lambda)=\lambda I-A, A \in \mathrm{C}^{n \times n}$, admits two distinct canonical factorizations. One is given by

$$
L(\lambda)=F(\lambda) D(\lambda) G(\lambda)
$$

where $F$ and $G$ are unimodular and $D=\operatorname{diag}\left(d_{1}, \ldots, d_{n}\right)$ is the Smith form of $L$. The other is

$$
L(\lambda)=C(\lambda I-J) B
$$

where $J$ is the Jordan form of $A, B=C^{-1}$, and the columns of $C$ are chains of eigenvectors and generalized eigenvectors of $A$. In the case of a general complex polynomial matrix $M \in \mathbf{C}^{n \times n}[\lambda]$,

$$
M(\lambda)=\sum_{\mu=0}^{m} A_{\mu} \lambda^{\mu}, \quad A_{\mu} \in \mathbf{C}^{n \times n},
$$

only a factorization of type (1.1) has been considered. It is the purpose of this note to show that $M$ too can be factored into a product of type (1.2),

$$
M(\lambda)=\hat{C}(\lambda)(\lambda I-J) \hat{B}(\lambda),
$$

provided $M$ has a proper rational inverse. The columns of $\hat{C}(\lambda)$ consist of eigenvectors and generalized eigenvectors of a linear operator associated with $M$. Our investigation uses Fuhrmann's approach [4] to the realization of rational matrices.

2. Jordan chains of polynomial matrices. We shall use the following notation. $\mathbf{C}^{n}[\lambda]$ will denote the space of all column vectors with complex polynomial entries. Similarly $\mathbf{C}^{n \times k}[\lambda]$ denotes the vector space of all $n \times k$ complex polynomial matrices. The entries of vectors in $\mathbf{C}^{n}(\lambda)$ and of matrices in $\mathbf{C}^{n \times k}(\lambda)$ are complex rational functions. A complex function $f$ is called

Received by the editors July 6, 1978.

AMS (MOS) subject classifications (1970). Primary 15A54, 15A18, 15A21; Secondary 93B99.

Key words and phrases. Polynomial matrices, Jordan chains, Jordan normal form, realizations, shift operator. 
proper rational, if it is the quotient of two polynomials, $f=p / q$, and $\operatorname{deg} p<$ deg $q$. An element of $C^{n}(\lambda)$ or $C^{n \times k}(\lambda)$ is proper rational if all of its entries are proper rational.

For the definitions and results of this section we refer to [1], [6], [7]. A number $\lambda_{0} \in \mathbf{C}$ is called a characteristic root of $M \in \mathbf{C}^{n \times n}[\lambda]$, if $\operatorname{det} M\left(\lambda_{0}\right)=$ 0. A Jordan chain of $M$ corresponding to the characteristic root $\lambda_{0}$ is a sequence of vectors $c_{1}, c_{2}, \ldots, c_{s}, c_{\sigma} \in \mathbf{C}^{n}$, which satisfy $c_{1} \neq 0$ and

$$
\sum_{p=0}^{j} \frac{1}{p !} M^{(p)}\left(\lambda_{0}\right) c_{j+1-p}=0, \quad j=0,1, \ldots, s-1 .
$$

The chain $c_{1}, \ldots, c_{s}$ of length $s$ will be called full, if it cannot be extended to a chain of length $s+1$. The sequence $c_{s}, c_{s-1}, \ldots, c_{1}$ is called a reversed chain. In the special case of $M(\lambda)=\lambda I-A, A \in C^{n \times n}$, the relations (2.1) yield

$$
\left(\lambda_{0} I-A\right) c_{1}=0, \quad\left(\lambda_{0} I-A\right) c_{i+1}=c_{i}, \quad i=1, \ldots, s-1,
$$

and a Jordan chain of $M$ consists of an eigenvector $c_{1}$ and generalized eigenvectors of $A$.

We shall use $J$ for a matrix in (upper triangular) Jordan form,

$$
J=\text { block } \operatorname{diag}\left(J_{1}, \ldots, J_{g}\right) \text {, }
$$

where

$$
J_{\gamma}=\left(\begin{array}{ccccc}
\lambda_{\gamma} & 1 & 0 & \cdots & 0 \\
0 & \lambda_{\gamma} & 1 & \cdots & 0 \\
\vdots & \vdots & \vdots & \ddots & \vdots \\
0 & \cdot & \cdot & \cdots & \lambda_{\gamma}
\end{array}\right) .
$$

The columns of a matrix $C \in \mathbf{C}^{n \times r}$ are said to form a full system [7] of Jordan chains of $M(\lambda)=\sum_{\mu=0}^{m} A_{\mu} \lambda^{\mu}$, if

$$
r=\operatorname{deg} \operatorname{det} M
$$

and if there is a matrix $J \in \mathbf{C}^{\times r}$ such that

$$
\sum_{\mu=0}^{m} A_{\mu} C J^{\mu}=0
$$

and

$$
\operatorname{rank}\left(\begin{array}{c}
C \\
C J \\
\vdots \\
C J^{r-1}
\end{array}\right)=r
$$

The conditions (2.3)-(2.5) are equivalent (see [7]) to the property that the columns of $\mathrm{Ce}^{\mathrm{Jt}}$ form a fundamental system of solutions of the differential 
equation

$$
\sum_{\mu=0}^{m} A_{\mu} x^{(\mu)}(t)=0 .
$$

If $J$ has the block structure (2.2), then $C$ can be partitioned into $C=$ $\left(C_{1}, \ldots, C_{g}\right)$ such that the columns of each $C_{\gamma}$ form a full chain. Analogously the columns of $B^{T}$ are said to form a full system of reversed chains of $M^{T}$ if $\sum J^{\mu} B A_{\mu}=0$ and

$$
\operatorname{rank}\left(B, J B, \ldots, J^{r-1} B\right)=r .
$$

In the case $M(\lambda)=\lambda I-A$ the preceding definitions yield nonsingular matrices $C$ and $B$ such that

$$
\lambda I-A=C(\lambda I-J) C^{-1}=B^{-1}(\lambda I-J) B .
$$

3. Realizations. Let $W \in \mathrm{C}^{k \times n}(\lambda)$ be a proper rational matrix. Thus $W$ has a formal expansion

$$
W(\lambda)=\sum_{\mu=0}^{\infty} W_{i} \lambda^{-(i+1)}
$$

LEMMA 1 [4]. There is an invertible matrix $M \in C^{k \times k}[\lambda]$ and a matrix $P \in \mathbf{C}^{k \times n}[\lambda]$ such that

$$
W=M^{-1} P
$$

and $M$ and $P$ are left coprime. $M$ and $P$ are unique up to a common left unimodular factor. Analogously there exist an invertible matrix $N \in \mathbf{C}^{n \times n}[\lambda]$ and a matrix $Q \in \mathrm{C}^{k \times n}[\lambda]$ such that

$$
W=Q N^{-1}
$$

and $N$ and $Q$ are right coprime.

Definition (see e.g. [2], [4]). A triple of linear operators $[A, B, C], B$ : $\mathrm{C}^{n} \rightarrow X, A: X \rightarrow X, C: X \rightarrow C^{k}$ is called a realization of $W$, if

$$
W_{i}=C A^{i} B, \quad i=0,1,2, \ldots
$$

The realization is called minimal, if the dimension of the state space $X$ is minimal.

We note the following result on realizations of $W$.

LEMMA 2 (see [3]). If $p$ is the least common denominator of all minors of $W$ and $r=\operatorname{deg} p$, then there is a minimal realization $[J, B, C]$ of $W$ with state space $X=\mathbf{C}^{\top}$ such that

$$
W(\lambda)=C(\lambda I-J)^{-1} B
$$

and $J \in \mathbf{C}^{\times r}$ is in Jordan form. For any minimal realization $J, B, C$ the conditions (2.5) and (2.6) hold and the characteristic polynomial of $J$ is equal to $p$.

Since (2.5) is equivalent to $\lambda I-J$ and $C$ being right coprime and (2.6) to 
$\lambda I-J$ and $B$ being left coprime, the last statement of Lemma 2 is contained in the following result.

LeMma 3 [3, p. 102]. If $P, Q$ and $S$ are polynomial matrices such that $S$ is left coprime to $P$ and right coprime to $Q$, then the least common denominator of all minors of $W=Q S^{-1} P$ is equal to det $S$.

The matrices $C$ and $B$ in (3.3) can be characterized in terms of Jordan chains of $M$ and $N$ in (3.1) and (3.2).

THEOREM 1. If $W$ has the representations

$$
W(\lambda)=C(\lambda I-J)^{-1} B=M^{-1}(\lambda) P(\lambda)=Q(\lambda) N^{-1}(\lambda)
$$

such that $[J, B, C]$ is a minimal realization and $M$ and $P($ resp. $N$ and $Q$ ) are left (resp. right) coprime, then the columns of $C$ form a full system of Jordan chains of $M$ and the columns of $B^{T}$ make up a full system of reversed chains of $N^{T}$.

Proof. From $M(\lambda)=\sum_{\mu=0}^{m} A_{\mu} \lambda^{\mu}$ and $(\lambda I-J)^{-1}=\sum_{\nu=0}^{\infty} J^{\nu} \lambda^{-(\nu+1)}$ the expansion of

$$
M(\lambda) C(\lambda I-J)^{-1} B
$$

into a formal power series can be obtained. Since the matrix (3.4) is equal to the polynomial matrix $P$, the coefficients of $\lambda^{-s}, s=1,2, \ldots$, vanish. Therefore

$$
\sum_{\mu=0}^{m} A_{\mu} C J^{\mu+\sigma} B=0, \quad \sigma=0,1,2, \ldots,
$$

and (2.6) yields $\sum_{\mu=0}^{m} A_{\mu} C J^{\mu}=0$. Hence $C$ satisfies the conditions (2.4) and (2.5). Lemma 3 implies (2.3). Analogous reasoning is valid for $B$.

COROLLARY [7]. If $M \in C^{n \times n}[\lambda]$ has a proper rational inverse and $[J, B, C]$ is a minimal realization of $M^{-1}$ such that

$$
M^{-1}(\lambda)=C(\lambda I-J)^{-1} B
$$

then the columns of $C$ (of $B)$ form a full system of (reversed) Jordan chains of $M\left(\right.$ of $\left.M^{T}\right)$.

Proof. The degree of the least common denominator of all minors of $M^{-1}$ is the degree of the determinant of $M$. The result is thus a consequence of Lemma 2 and the preceding theorem.

4. The factorization theorem. For a nonsingular polynomial matrix $M \in$ $\mathrm{C}^{n \times n}[\lambda]$ the quotient module $Y=\mathbf{C}^{n}[\lambda] / M \mathbf{C}^{n}[\lambda]$ is a $\left.\mathrm{C} \lambda\right]$ module and also a vector space of dimension $r$ over $\mathrm{C}$, if $r=\operatorname{deg} \operatorname{det} M$. The module $Y$ is important in linear system theory. We follow Fuhrmann [4] in describing a concrete representation of $Y$ and defining a restricted shift operator in $Y$. Each $n$-vector of rational functions $f \in \mathbf{C}^{n}(\lambda)$ decomposes uniquely into a sum $f=g+h$ where the elements of $g$ are proper rational and $h \in C^{n}[\lambda]$. 
Define $\pi: \mathbf{C}^{n}(\lambda) \rightarrow \mathbf{C}^{n}(\lambda)$ to be the map which discards the polynomial part $h$ of $f, \pi f=g$. For $f \in C^{n}[\lambda]$ put

$$
\pi_{M} f=M \pi\left(M^{-1} f\right) .
$$

Then $\pi_{M}: \mathbf{C}^{n}[\lambda] \rightarrow \mathbf{C}^{n}[\lambda]$ is a projection map and ker $\pi_{M}=M \mathbf{C}^{n}[\lambda]$. Thus $K_{M}:=\operatorname{Im} \pi_{M}$, the image of $\pi_{M}$, is a $\mathrm{C}[\lambda]$ module isomorphic to $Y$. The shift operator $S(M)$ restricted to $K_{M}$ is defined by

$$
S(M) f(\lambda)=\pi_{M} \lambda f(\lambda), \quad f \in K_{M} .
$$

LEMMA 4 [4]. The eigenvalues of $S(M)$ are the characteristic roots of $M$. The eigenvectors of $S(M)$ corresponding to an eigenvalue $\lambda_{0}$ have the form $\lambda-$ $\left.\lambda_{0}\right)^{-1} M(\lambda) \xi$ and $M\left(\lambda_{0}\right) \xi=0$.

We establish a basis of $K_{M}$ which exhibits $S(M)$ in Jordan form.

THEOREM 2. If the columns of $C$ are a full system of Jordan chains of $M$, then the columns of the polynomial matrix

$$
\hat{C}(\lambda)=M(\lambda) C(\lambda I-J)^{-1}
$$

form a basis of $K_{M}$ such that

$$
S(M) \hat{C}(\lambda)=\hat{C}(\lambda) J
$$

Proof. If we extend the definition of $\pi$ in a natural way from $\mathbf{C}^{n}(\lambda)$ to rational matrices in $\mathbf{C}^{n \times k}(\lambda)$, then

$$
\pi_{M} \hat{C}(\lambda)=M(\lambda) \pi C(\lambda I-J)^{-1}=\hat{C}(\lambda) .
$$

Therefore the columns of $\hat{C}(\lambda)$ are in $K_{M}$, and because of (2.5) and $M$ nonsingular they are linearly independent. From $\pi \lambda(\lambda I-J)^{-1}=(\lambda I-$ $J)^{-1} J$ we obtain

$$
S(M) \hat{C}(\lambda)=M(\lambda) C \pi \lambda(\lambda I-J)^{-1}=\hat{C}(\lambda) J .
$$

Analogously $\hat{B}(\lambda)$, defined by

$$
\hat{B}(\lambda)=(\lambda I-J)^{-1} B M(\lambda)
$$

is a polynomial matrix and the columns of $\hat{B}(\lambda)^{T}$ form a basis of $K_{M^{T}}$ and $S\left(M^{T}\right) \hat{B}(\lambda)^{T}=\hat{B}(\lambda)^{T} J^{T}$.

The preceding definitions of $\hat{C}(\lambda)$ and $\hat{B}(\lambda)$ lead to the factorization result.

THEOREM 3. Let $M \in \mathrm{C}^{n \times n}[\lambda]$ be a matrix which has a proper rational inverse and let $M^{-1}$ have a minimal realization $[J, B, C]$,

$$
M^{-1}(\lambda)=C(\lambda I-J)^{-1} B
$$

such that $J$ is an $r \times r$ matrix in Jordan form. Then

$$
M(\lambda)=\hat{C}(\lambda)(\lambda I-J) \hat{B}(\lambda)
$$

where $\hat{B}(\lambda)$ and $\hat{C}(\lambda)$ are defined by (4.1) and (4.2).

REMARK. In the special case of $M(\lambda)=\lambda I-A$ the theorem yields the transformation of $A$ into Jordan normal form, that is $\lambda I-A=C(\lambda I-J) B$, $B=C^{-1}$. 
In the sequel of this note we shall study the case where $M$ does not have a proper rational inverse and the more general one of $M$ being rectangular. It will be shown that a factorization of the form

$$
M(\lambda)=\hat{C}(\lambda)\left(\lambda A_{1}+A_{2}\right) \hat{B}(\lambda)
$$

is possible and that Kronecker's theory of matrix pencils [5] can be extended to rectangular polynomial matrices. In the special case of a unimodular matrix $M,(4.3)$ leads to $M(\lambda)=\hat{C}(\lambda)(\lambda R+I) \hat{B}(\lambda)$ where $R$ is nilpotent.

\section{REFERENCES}

1. H. Baumgärtel, Endlichdimensionale analytische Störungstheorie, Akademie Verlag, Berlin, 1972.

2. R. W. Brockett, Finite dimensional linear systems, Wiley, New York, 1970.

3. W. A. Coppel, Matrices of rational functions, Bull. Austral. Math. Soc. 11 (1974), 89-113.

4. P. Fuhrmann, Algebraic system theory, an analyst's point of view, J. Franklin Inst. 301 (1976), 521-540.

5. F. R. Gantmacher, Matrizenrechnung, Teil II, 3. Aufl., VEB Deutscher Verlag der Wissenschaften, Berlin, 1971.

6. P. Lancaster and H. K. Wimmer, Zur Theorie der $\lambda$-Matrizen, Math. Nachr. 68 (1975), 325-330.

7. H. K. Wimmer, Jordan-Ketten und Realisienungen rationaler Matrizen, Linear Algebra and Appl. 20 (1978), 101-110.

Mathiamatisches Institut, Universttät WÜrzburg, D8700 Würzburg, Federal Republic OF Germany 